\title{
Spatial bifurcations of fixed points and limit cycles during the electrochemical oxidation of $\mathrm{H}_{2}$ on Pt ring-electrodes
}

\author{
Peter Grauel, Hamilton Varela and Katharina Krischer* \\ Fritz-Haber-Institut der Max-Planck-Gesellschaft, Faradayweg 4-6, 14195 Berlin, \\ Germany.E-mail: krischer@fhi-berlin.mpg.de
}

Received 12th April 2001

First published as an Advance Article on the web 12th November 2001

Pattern formation during the oscillatory oxidation of $\mathrm{H}_{2}$ on $\mathrm{Pt}$ ring-electrodes in the presence of electrosorbing ions was studied under potentiostatic control for three different positions of the reference electrode (RE). The position of the RE crucially affects the degree of the global feedback which is imposed by the potentiostatic operation mode, and the three configurations selected corresponded to zero, maximum and intermediate global coupling. In the absence of global coupling, 'communication' among different positions occurs exclusively through migration coupling (the electrochemical counterpart to diffusion in reaction-diffusion systems). In this case, spatially inhomogeneous oscillations that were attributed to a spatial bifurcation of the homogeneous limit cycle were observed throughout. This implies that the system is Benjamin-Feir unstable. For the strongest global coupling adjustable, travelling pulses were found that emerged in a wave bifurcation with $n=1$ from the homogeneous steady state. The pulses exhibited modulations in velocity and width that most likely resulted from the interaction between inhomogeneities of the catalytic surface and the nonlinear reaction dynamics. In the case of an intermediate global coupling strength, a diversity of spatio-temporal motions was observed. The dynamics ranged from pulses over target patterns and so-called asymmetric target patterns to mixed states where two or three of these states alternate. For some parameters these mixed states were in addition separated by bursts of the system to a nearly homogeneous unreactive state.

\section{Introduction}

The vast majority of electrochemical systems undergo dynamic instabilities resulting, e.g., in sustained temporal oscillations, bistability or spatio-temporal patterns (for recent review articles see ref. 1-3). In general, these phenomena are caused by the interaction between nonlinear electrode kinetics and electric properties of the entire system, most importantly the potential drop through the electrolyte. This second property makes pattern formation in electrochemical systems extremely sensitive to the relative arrangement of working electrode (WE), counter electrode (CE) and reference electrode (RE). In particular, it facilitates experimental studies of pattern formation in the presence of various spatial couplings.

To make this clear, let us review the mechanism of coupling among reacting sites in electrochemical systems. The central variable for pattern formation in electrode reactions is the potential drop across the double layer, $\phi_{\mathrm{DL}}$, and different locations of the electrode are coupled through the electric field in the electrolyte. Thus potential changes at some location of the WE are felt rapidly 
at other locations. The coupling is non-local because its effect is felt by a whole neighbouring range (in some geometries by the whole electrode) but with decreasing strength for increasing distance.

The electric field or, equivalently, the potential distribution in the electrolyte is determined mainly by the potential of the WE and CE and the relative locations of these electrodes. Since the $\mathrm{CE}$ constitutes an equipotential plane, a pattern in $\phi_{\mathrm{DL}}$ that establishes at the WE exists all over the electrolyte up to the CE, though the pattern smooths and its amplitude diminishes with increasing distance from the WE. In the following, we only consider situations in which the WE and the CE are parallel to each other. Then, the distance between the two electrodes determines the range of the coupling. It is maximum if this distance is much larger than the lateral extension of the working electrode and becomes more localised for smaller distances. This coupling through the electric field is always effective in an electrochemical experiment, and is referred to as migration coupling. Thus, in electrochemical systems migration coupling takes the role of diffusion coupling in reaction-diffusion systems.

In a potentiostatic experiment, the voltage between the WE and the RE is kept constant. In general, the RE 'sees' the potential at a certain location in the electrolyte. If this location is behind the CE (see e.g. the external RE in Fig. 1), then it is located in an equipotential plane parallel to the electrode, and the coupling remains unaffected by the RE. If, on the other hand, it is located between the WE and the CE, e.g. to minimize the voltage drop through the electrolyte (see $\mathrm{RE}(2)$ in Fig. 1), the potential at the position of the RE is altered by any local change of the double layer potential (because the latter affects the potential distribution in the entire electrolyte up to the $\mathrm{CE}$ ). As a consequence, the actual voltage between the WE and the RE differs from the set voltage, and the potentiostat changes the potential of the WE. In other words, charge is pumped into the entire double layer. If the distance between every position of the WE and the RE is equal, the potential at the position of the RE is a function of the average double layer potential, and the feedback by the potentiostat imposes a global coupling on the system. The strength of the coup-

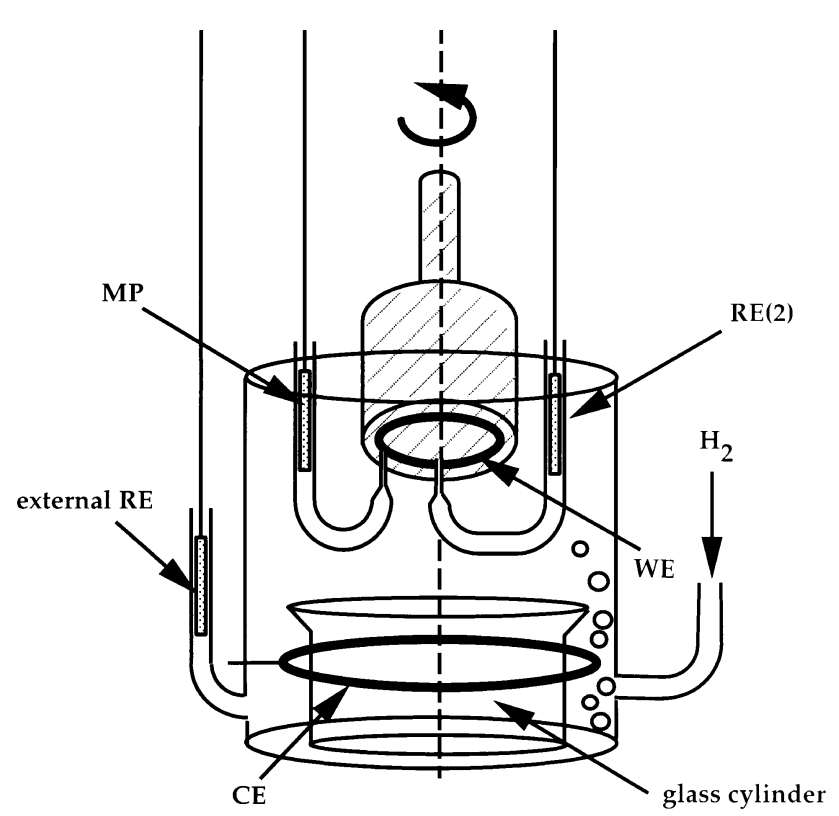

Fig. 1 Schematic view of the experimental set-up. WE: working electrode, a rotating Pt ring-electrode embedded into a Teflon body, RE: reference electrode. Two types of RE were used: (1) a $\mathrm{Hg} / \mathrm{Hg}_{2} \mathrm{SO}_{4}$ electrode in a separate compartment (external RE) and (2) a $\mathrm{Ag} / \mathrm{AgCl}$ electrode $(\mathrm{RE}(2))$ put on the axis of the ring and close to the WE with the help of a glass (Haber-Luggin) capillary. In one set of experiments the distance between $\mathrm{RE}(2)$ and the plane of the WE was $3 \mathrm{~mm}$, in another set it was $13 \mathrm{~mm}$. CE: counter electrode, a Pt ring. MP: potential micro-probe. 
ling is a function of the distance between the WE and the RE: The closer the WE and the RE are, the stronger is the global coupling.

In summary, the distance between the WE and the CE determines the nonlocality of the migration coupling. The distance between the WE and the RE determines the strength of an additional global coupling.

Global constraints exist in a variety of physical and chemical systems. Among them are catalytic reactors, in which the external constraint stems from the electric control of the temperature $^{4-6}$ or in which the global coupling is due to fast mixing of the gas phase, ${ }^{7,8}$ gasdischarge devices, ${ }^{9}$ semiconductor systems ${ }^{10}$ or ferromagnetic systems. ${ }^{11}$ Experimental and theoretical studies of these systems revealed a rich plethora of spatiotemporal patterns (see, e.g. ref. 6, 9 and 12-24).

Concerning electrochemical systems, there are some examples demonstrating the impact of global coupling on pattern formation in the bistable and the oscillatory region. The pioneering experiment goes back to Otterstedt et al. who observed pulses travelling around Co electrodes during electrodissolution of the electrode. ${ }^{25}$ In later studies these authors also report more complex motions in the same system. ${ }^{26,27}$ Stationary domains were found during the reduction of persulfate ${ }^{28}$ and the electrooxidation of $\mathrm{H}_{2} \cdot{ }^{3,29}$ Standing waves formed during formic acid oxidation when a close reference electrode was used. ${ }^{30}$ In the bistable region of this reaction, fronts could be remotely triggered, ${ }^{31}$ another peculiar manifestation of global coupling. The difference between the impact of global coupling in electrochemical systems with an N- and an S-shaped current potential curve is discussed in ref. 32 .

In this contribution we extend these studies on the impact of global coupling on patterns in electrochemical systems. The system under consideration is the oscillatory oxidation of $\mathrm{H}_{2}$ on $\mathrm{Pt}$ ring-electrodes. By adjusting three different positions of the RE, the strength of the global coupling is varied. In the first set of experiments the RE is located behind the CE. Thus, this system can be viewed as a 'reference system' without global coupling. In the second set of experiments, the $\mathrm{RE}$ is placed on the axis of the WE at approximately the closest distance that can be adjusted. Finally, an intermediate distance between the WE and the RE, and hence also an intermediate strength of the global coupling is realised. Besides some of the already known manifestations of global coupling, other novel wave types are reported.

\section{Experimental}

A schematic view of the experimental set-up is shown in Fig. 1. A rotating Pt ring (outer diameter $30 \mathrm{~mm}$, width $1 \mathrm{~mm}$ ) embedded into a Teflon cylinder served as the working electrode (WE). Two different configurations were used for the reference electrode (RE). Either a $\mathrm{Ag} / \mathrm{AgCl}$ electrode $(\mathrm{RE}(2))$ was put into a J-shaped glass capillary (Haber-Luggin capillary) whose tip was located on the axis of the WE and adjusted at two different distances (namely 3 and $13 \mathrm{~mm} \pm 0.2 \mathrm{~mm}$ ) to the plane of the WE, or a $\mathrm{Hg} / \mathrm{Hg}_{2} \mathrm{SO}_{4}$ reference electrode in a separate compartment (external RE) that was connected to the main compartment below the plane of the CE was used. The CE, a 1 $\mathrm{mm}$ thick Pt wire bent to a ring of $65 \mathrm{~mm}$ in diameter, was located parallel and at a distance of 45 $\mathrm{mm}$ to the WE.

To monitor the angular potential distribution in front of the WE, the tip of a second glass capillary equipped with an $\mathrm{Ag} / \mathrm{AgCl}$ electrode (the potential micro-probe, $\mathrm{MP}$ ) was placed 1 $\mathrm{mm} \pm 0.2 \mathrm{~mm}$ below the Pt ring (WE). During the experiments the WE was rotated at $20 \mathrm{~Hz}$, and the voltage between the MP and the WE was measured with an acquisition rate of $1 \mathrm{kHz}$ (i.e. 50 points/rotation), which allowed us to construct a spatiotemporal picture of the potential in front of the WE. As the resistance between the MP and the WE was negligible, the measured voltage represents the local potential drop across the double layer (called double layer potential, $\phi_{\mathrm{DL}}$, below) to a good approximation. For further experimental details see ref. 29 and 33.

The chemicals, $\mathrm{H}_{2}\left(5 \mathrm{~N}\right.$, Linde), $\mathrm{H}_{2} \mathrm{SO}_{4}$ (p.a., Merck), $\mathrm{HCl}$ (p.a., Merck) and $\mathrm{CuSO}_{4}$ (p.a., Merck) were used as received and solutions were prepared from millipore water (suprapure system, MilliQ). The electrolyte used in all experiments consisted of $0.5 \mathrm{mM} \mathrm{H}_{2} \mathrm{SO}_{4}, 0.1 \mathrm{mM} \mathrm{HCl}$ and 0.025 $\mathrm{mM} \mathrm{CuSO}{ }_{4}$ saturated with $\mathrm{H}_{2}$, which was continuously bubbled through the cell during the experiments. 
Prior to each experiment the base electrolyte $\left(0.5 \mathrm{mM} \mathrm{H}_{2} \mathrm{SO}_{4}\right)$ was purged with $\mathrm{N}_{2}$ in order to remove dissolved oxygen. Then, the $\mathrm{Pt}$ working electrode was electrochemically cleaned through fast voltammetric scans for $1 \mathrm{~h}$ between the potentials at which hydrogen and oxygen evolution set in. Afterwards, the electrolyte was saturated with $\mathrm{H}_{2}$, and $\mathrm{HCl}$ and $\mathrm{CuSO}_{4}$ were added. The dynamic behavior was investigated under potentiostatic conditions, i.e. during one experiment the voltage between the WE and the RE (either the external RE or RE(2), vide supra), $U$, was kept constant by means of a potentiostat (FHI electronic laboratory).

Concomitant to the $\mathrm{H}_{2}$ oxidation at the working electrode, $\mathrm{Cu}$ was deposited on the $\mathrm{CE}$. Consequently, the $\mathrm{Cu}$ concentration changed slightly on the timescale of hours, being somewhat lower at the end of the experiment than in the beginning. However, the change in $\mathrm{Cu}$ concentration was slow compared to the characteristic time (e.g. the oscillation periods) of the phenomena studied. Thus, in the time window in which a certain time series was recorded the parameters of the system were constant to a very good approximation. The long-time drift in the $\mathrm{Cu}$ concentration resulted in the same dynamic behavior being found at somewhat shifted values of the external potential $U$ when repeating the experiment after an hour. Also, on different days, the quantitative values of $U$ at which a certain behavior was found varied somewhat, most likely due to slight differences in the roughness of the surface. But the qualitative dynamics, i.e. the types and sequences of dynamical states observed, were well reproducible.

\section{Results}

In the following we describe pattern formation during the oscillatory $\mathrm{H}_{2}$ oxidation as a function of the external potential for the three different positions of the RE used. Firstly, the external RE is considered, and thus the case without global coupling. Then, we investigate the behavior for the closest distance between the WE and the RE, and thus the strongest coupling that can be adjusted. Finally we look at the dynamics in the case of intermediate coupling strength.

\subsection{External reference electrode}

In Fig. 2 a current-voltage $(I / U)$ curve recorded during a potential scan in the positive direction is displayed. The curve represents a rough picture of the global behavior that is also found under stationary conditions. At low values of the applied potential a stable stationary fixed point exists which undergoes an instability that gives way to large amplitude oscillations. Directly beyond the bifurcation, the oscillations possess a very long period whereby the current remains quasistationary close to the former fixed point for most of the oscillation period. From this quasistationary state the trajectory spirals out exhibiting high frequency oscillations with small but slowly increasing amplitude ( $c f$. Fig. 3a) until the system undergoes a large excursion to high values of the current density from where it returns back to the quasistationary state. This behavior suggests that the oscillations are born in a homoclinic bifurcation. For increasing external voltage

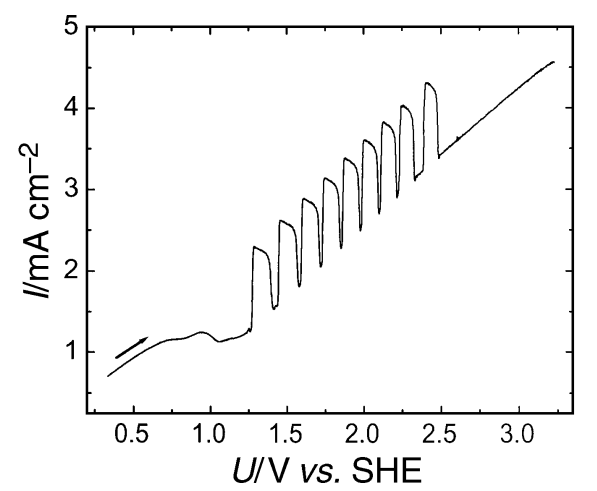

Fig. $2 I / U$ potentiodynamic profile of a rotating $\mathrm{Pt}$ ring-electrode during the $\mathrm{H}_{2}$ oxidation reaction in 0.5 $\mathrm{mM} \mathrm{H}_{2} \mathrm{SO}_{4}$ solution in the presence of $0.025 \mathrm{mM} \mathrm{CuSO}_{4}$ and $0.1 \mathrm{mM} \mathrm{HCl}$; rotation speed of the electrode: $20 \mathrm{~Hz}$; scan rate: $50 \mathrm{mV} \mathrm{s}^{-1}$. The external reference electrode was used, scan region: 0.33 to $2.23 \mathrm{~V}$. 

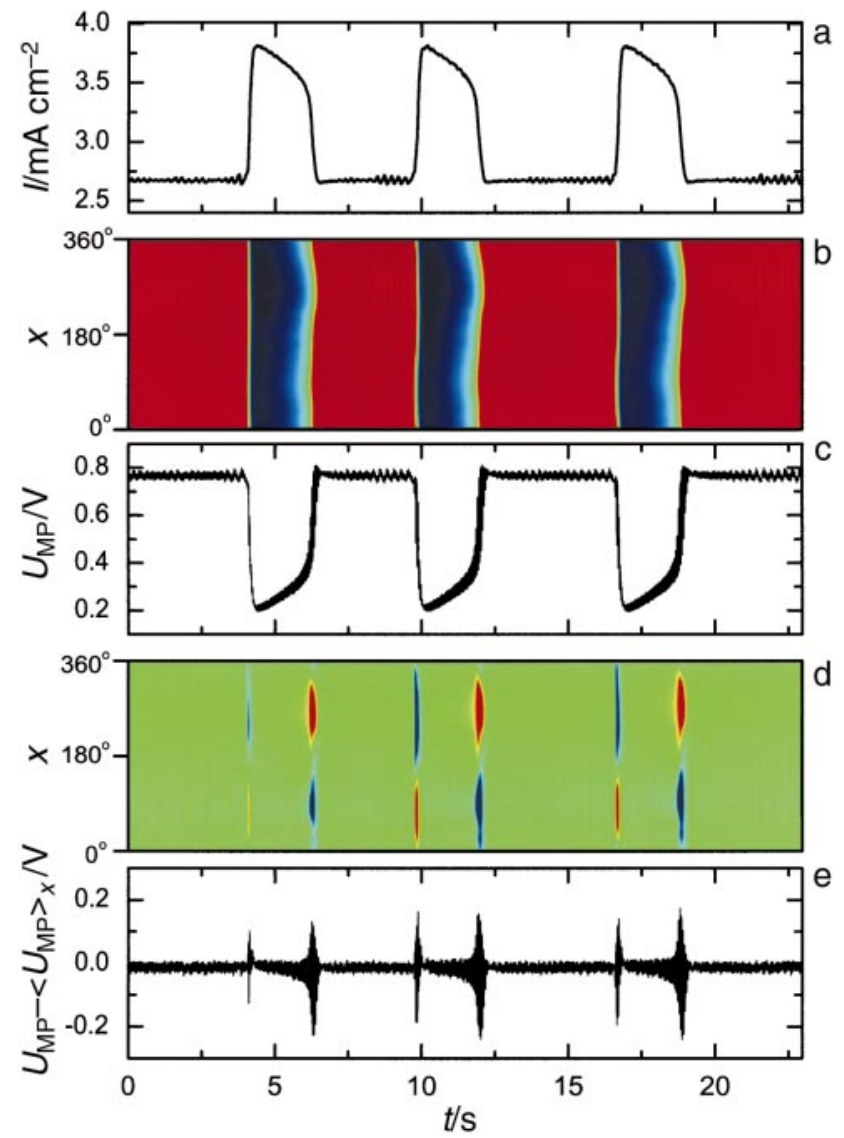

Fig. 3 Spatio-temporal dynamics in the oscillatory region under potentiostatic conditions with the external RE. External voltage $U: 2.05 \mathrm{~V} v$ s. SHE, other conditions as in Fig. 2. (a) Time series of the global current. (b) Position-time plot of the local double layer potential as measured with the potential micro-probe $\left(U_{\mathrm{MP}}(x, t)\right)$. In the rainbow colour scale red denotes the highest potential value and blue the lowest one. (c) Plot of the micro-probe potential $\left(U_{\mathrm{MP}}(x, t)\right)$ as a function of time. (d) Position-time plot of the inhomogeneously oscillating part of (b) obtained by subtraction of the homogeneous part from the micro-probe potential $\left(U_{\mathrm{MP}}(x, t)\right.$ $-\left\langle U_{\mathbf{M P}}(t)\right\rangle_{x}$ ). (e) Plot of the inhomogeneously oscillating part of the micro-probe potential as a function of time.

the oscillation period becomes shorter, while the amplitude stays approximately constant up to the high potential limit of the oscillations where the limit cycle disappears in a second hard bifurcation and the behavior becomes stationary again.

A typical space-time measurement in the oscillatory region at a constant value of the external potential $U$ is displayed in Fig. 3. Fig. 3a shows the time series of the global current and Fig. 3b the double layer potential as a function of space and time. At first glance, the oscillations appear to be homogeneous. However, when investigating the plot more carefully, in the transition region from high to low current a wavy structure of the double layer potential is clearly discernible. The inhomogeneous character of the oscillations becomes much better visible when subtracting at each instant in time the spatial average from the data (Fig. 3d). In this representation it is obvious that both flanks of the relaxation-type global oscillation are accompanied by a spatial symmetry breaking. The inhomogeneous part of the oscillation can be approximated as an oscillating sinusoidal structure of wavenumber one whereby the sign of the amplitude is different on the two flanks of the oscillation.

Fig. $3 \mathrm{c}$ and e show the time series of the micro-probe signal, $U_{\mathbf{M P}}(\mathrm{c})$ and the micro-probe signal after subtracting the spatial average, $U_{\mathbf{M P}}-\left\langle U_{\mathbf{M P}}\right\rangle_{x}(\mathrm{e})$, i.e. the same data of Fig. $3 \mathrm{~b}$ and $\mathrm{d}$ in a 
different representation. In these plots the absolute values of the double layer potential as well as the amplitudes of the homogeneous and inhomogeneous oscillations can be easily read off. The homogeneous oscillation possesses an amplitude of about $600 \mathrm{mV}$, the inhomogeneous structure having a maximal amplitude of about $300 \mathrm{mV}$.

Qualitatively the same spatio-temporal behavior was observed over the entire oscillatory region; an inhomogeneous structure, close to a sinusoidal mode with wavenumber one was always excited on the flanks of the relaxation type oscillations of the total current.

\subsection{Small distance between RE and WE}

When minimizing the distance between the RE and the WE, i.e. when using a Haber-Luggin capillary $(\mathrm{RE}(2))$ and adjusting a distance of $3 \mathrm{~mm}$ between the tip of the capillary and the plane of the working electrode, the spatio-temporal dynamics was qualitatively different. The total current exhibited relatively harmonic oscillations of small amplitude (Fig. 4). At the low potential end, the oscillations looked as if they were associated with a period-doubled limit cycle (Fig. 4a-c). For increasingly positive potential the difference between successive minima became smaller so that the current eventually exhibited period-1 oscillations (Fig. $4 \mathrm{~d}-\mathrm{f}$ ).

Space-time plots of the local double layer potential, $U_{\mathbf{M P}}(x, t)$ (without subtraction of the spatial average) corresponding to the time series of Fig. $4 \mathrm{a}$ and e are displayed in Fig. 5a and c. Obviously, this time we are dealing in the first place with a spatial symmetry breaking, namely a pulse that propagates around the ring in a fairly uniform manner. The oscillations in the current density

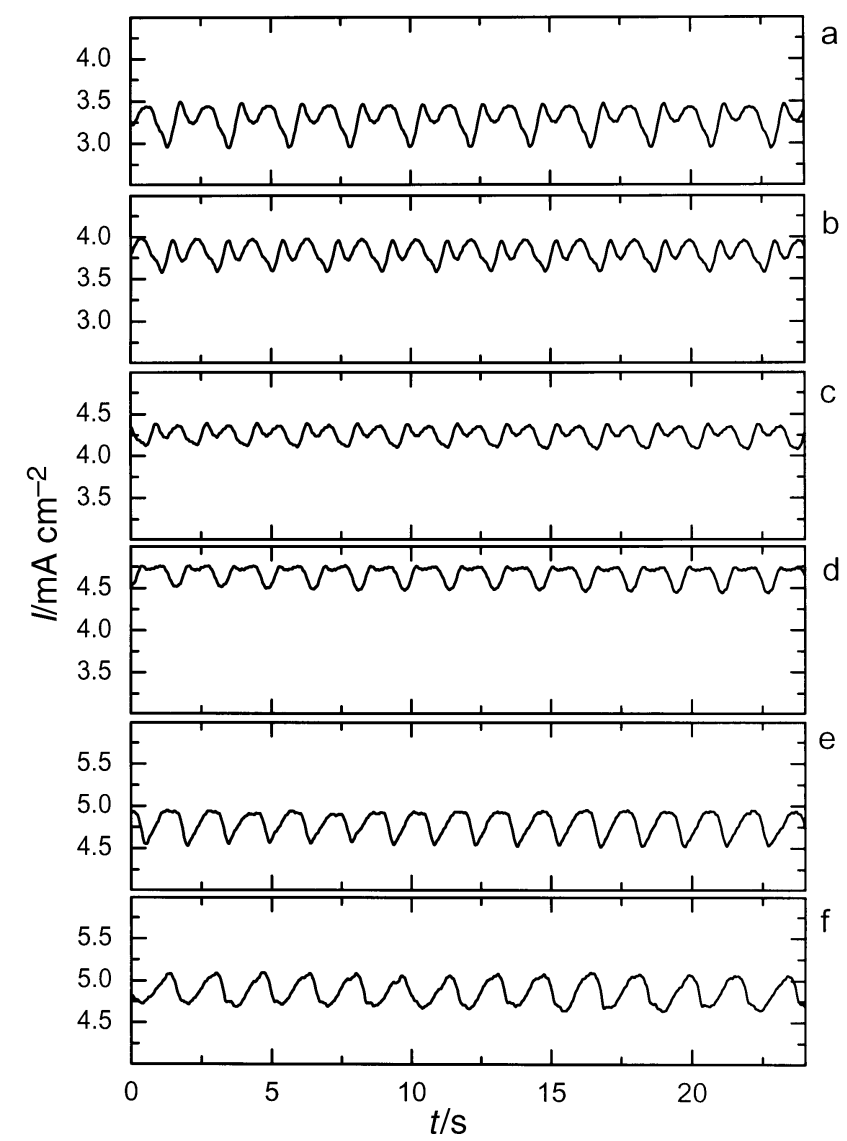

Fig. 4 Global current oscillations under potentiostatic conditions with the reference electrode $(\operatorname{RE}(2))$ placed at a distance of $3 \mathrm{~mm}$ from the plane of the WE for different values of the external voltage $U$ : (a) 0.96 , (b) 1.06 , (c) 1.16, (d) 1.26, (e) 1.36 and (f) $1.46 \mathrm{~V} v$ s. SHE. Other conditions as in Fig. 2. 


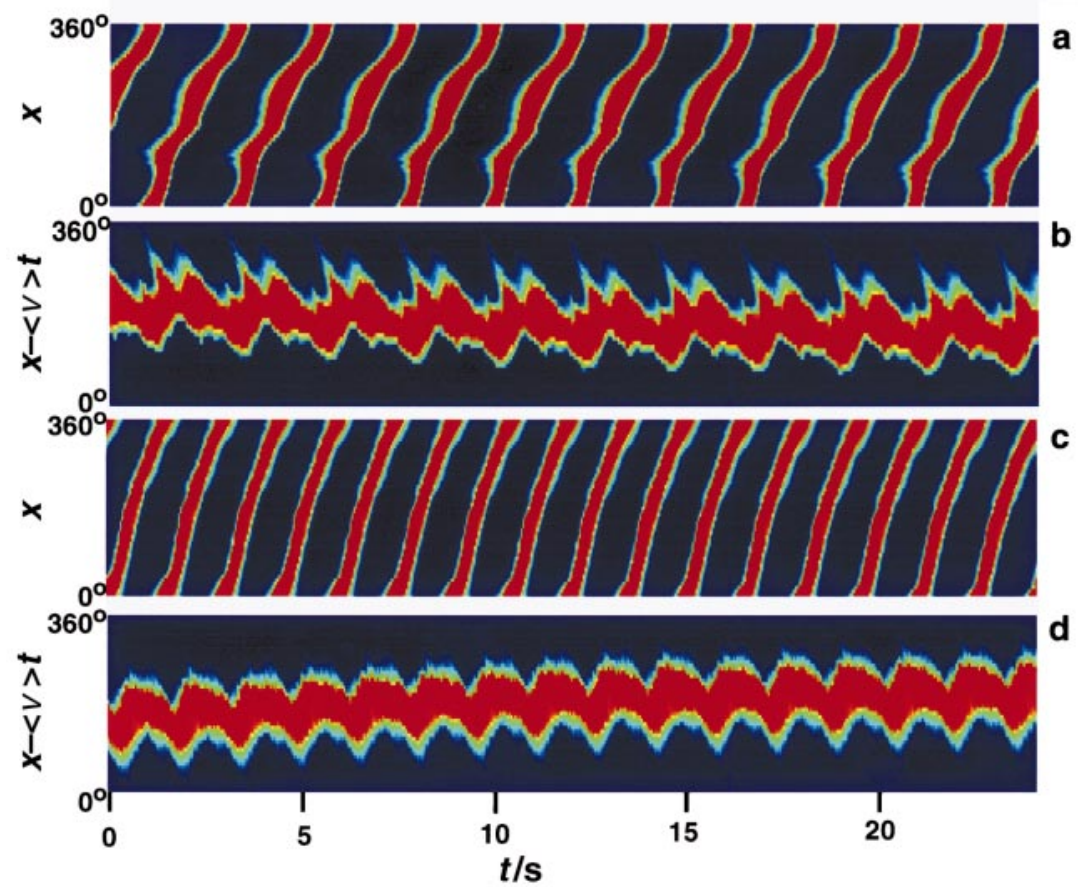

Fig. 5 Position-time plots of the local electrode potential $\left(U_{\mathrm{MP}}\right)$ in the laboratory coordinate system $((\mathrm{a})$ and (c)) and in a coordinate system moving with the mean velocity of the pulse ((b) and (d)) for the time series shown in Fig. 4(a) and (e).

arise as a result of changes in the width of the pulse during one rotation around the ring. This can be seen more clearly in Fig. $5 b$ and $d$ where the spatio-temporal behavior that one would observe in a coordinate system that moves with the mean velocity of the pulse is plotted. In the case of the simple periodic time series (Fig. 5d) the resulting picture is a 'zigzag' motion of the pulse, which exhibits some minor variations of the width. The latter cause the oscillations seen in the total current density (Fig. 4e), which possess the same period as the rotation period of the pulse, while the zigzag pattern indicates that the velocity of the pulse changes during one rotation. Not only is the back-and-forth motion of the pulse in the moving frame more pronounced in the first case (Fig. 5b), but the width of the pulse also oscillates more strongly and with twice the rotation period. In addition, a second active area is periodically excited at a certain position of the ring. (cf. the light blue-greenish offshoots in the upper part of the plot). This satellite pulse propagates quickly toward the main pulse and merges with the latter soon after its birth.

At this close distance between working and reference electrode, we never observed homogeneous oscillations. Rather, one of the two types of pulses emerged whenever the system did not attain a homogeneous stationary state. The mean pulse width and velocity increased with increasing $U$, the mean velocity ranging from $c a .4 \mathrm{~cm} \mathrm{~s}^{-1}$ (Fig. 4a) to $6.2 \mathrm{~cm} \mathrm{~s}^{-1}$ (Fig. 4e). Only very close to the end of the existence range of the pulses did the mean velocity decrease again slightly, e.g. in the case of Fig. $4 \mathrm{f}$ it amounts to $c a .5 .5 \mathrm{~cm} \mathrm{~s}^{-1}$.

\subsection{Intermediate distance between $\mathrm{RE}$ and WE}

The dynamics were by far richer for the intermediate distance between working and reference electrodes. An overview of the behavior is displayed in Fig. 6 where space-time plots of the double layer potential are shown together with the time series of the global current. The measurements in Fig. $6 \mathrm{a}-\mathrm{f}$ were obtained for increasing values of the external voltage. At low values of the external voltage the most complex spatio-temporal patterns were observed (Fig. 6a). A closer view suggests that it is made up of a sequence of behaviors that appear 'isolated' at larger values of $U$, which we therefore describe first. 

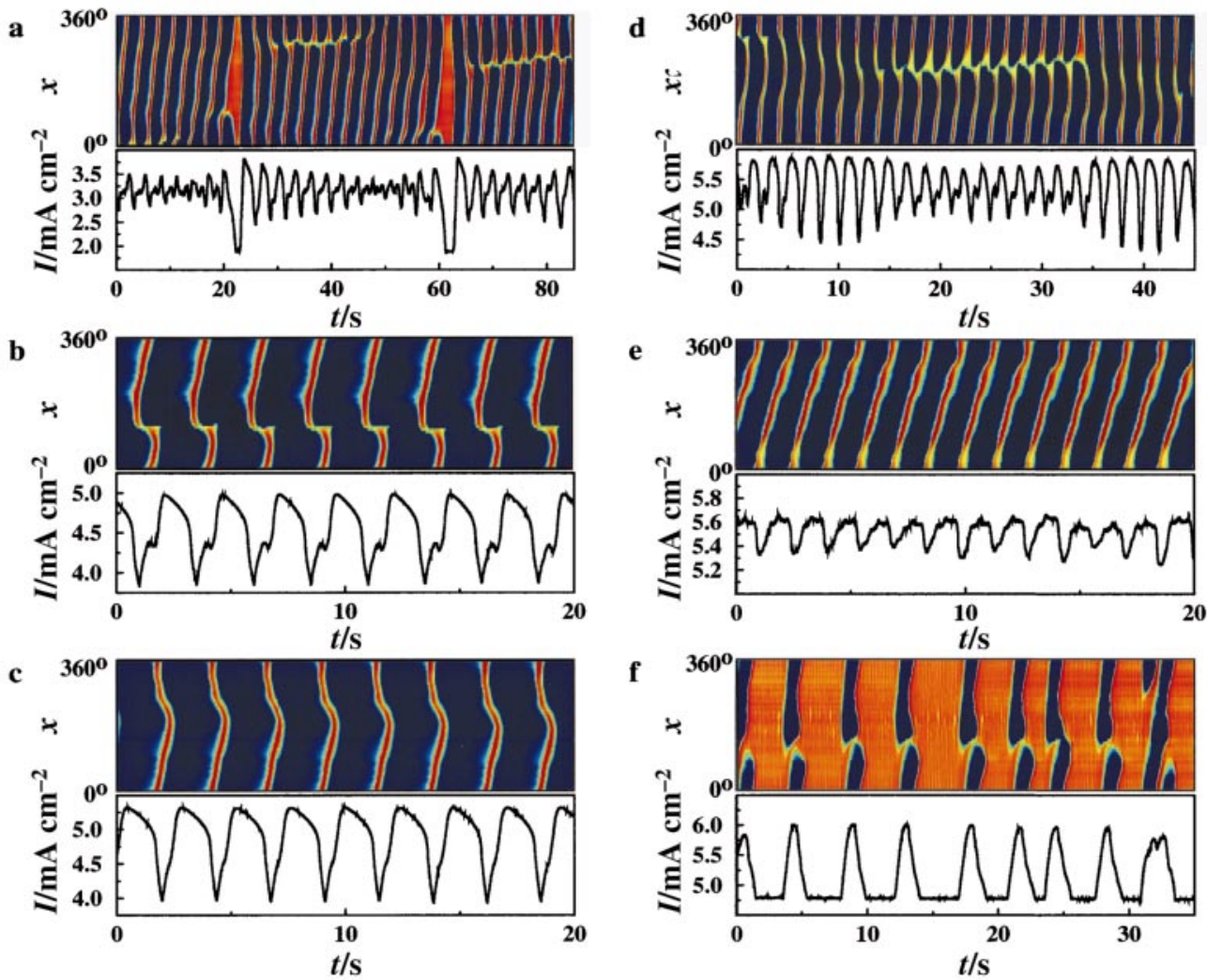

Fig. 6 Position-time plots of the local double layer potential and corresponding time series of the global current under potentiostatic conditions with the reference electrode $(\operatorname{RE}(2))$ placed at a distance of $13 \mathrm{~mm}$ from the plane of the WE for different values of the external voltage $U$ : (a) 1.06, (b) 1.37, (c) 1.5, (d) 1.67, (e) 1.76 and (f) $1.86 \mathrm{~V} v \mathrm{~s}$. SHE.

In Fig. 6e we recognize again a pulse travelling around the electrode with slightly changing velocity, similar to that shown in Fig. 5c.

The next simplest behavior appears to be that displayed in Fig. $6 \mathrm{c}$ : Position $0^{\circ}$ (or equivalently $360^{\circ}$ ) seems to be a wave source which periodically sends out two pulses that travel in opposite directions and meet in the center of the ring where they annihilate each other. From a phenomenological point of view, the pattern looks like the one-dimensional analogue of a 'classical' twodimensional target pattern. Following Zhabotinsky et al., ${ }^{34}$ who obtained similar dynamics in simulations of a three-component reaction-diffusion system, we also refer to it as target pattern. Note, however, that neither in those simulations nor in our experiments are the patterns linked to a pacemaker or to an excitable medium.

The spatio-temporal plot shown in Fig. $6 \mathrm{~b}$ resembles that of Fig. $6 \mathrm{c}$ in so far as there is also a source point that periodically emits waves. However, in this case the 'excitation' propagates only in one direction. The pulse that is about to travel into the opposite direction soon comes to a halt. The position at which the propagation of this second front is stopped remains in a more unreactive 'red state' until the propagating wave hits it. Upon this collision, both front and 'point excitation' are annihilated and the entire domain relaxes back to the 'blue' ground state until the cycle starts anew. In the following we call this behavior, which to our knowledge has not been described in the literature, an asymmetric target pattern.

In Fig. 6d we come across a mixture of the last two discussed behaviors: The system seems to switch back-and-forth between a more or less deformed variant of the target pattern, and the asymmetric target pattern. The complex overall behavior is also reflected in the time series of the 
total current density, which is apparently quasiperiodic. However, to determine whether we are indeed dealing with a quasiperiodic state requires much longer time series than we have recorded so far. The further characterization of the dynamics will be done in future work.

In Fig. 6a we meet a still higher degree of complexity. Four types of behavior take turns: Slightly imperfect target patterns are followed firstly by asymmetric target patterns, then by pulses and finally by the expansion of the less reactive portion of the pulse over the entire electrode before the scenario starts again. In the time series of the total current the global deactivation manifests itself in a large excursion toward smaller values. These large amplitude oscillations are separated by apparently quasiperiodic sections of the time series with smaller amplitudes that resemble the time series of Fig. 6d. Again, due to the complexity of the behavior which requires much longer measurements, that we have not yet obtained for technical reasons, we are currently not able to classify the behavior further, e.g. as periodic, quasi-periodic or chaotic. Note that the point-oscillation at which the front is extinguished in the asymmetric target pattern appears each time at a different position. The same is true for the measurement of Fig. 6d. This makes it very unlikely that structural defects of the electrode surface are responsible for the emergence of the asymmetric target patterns.

At the highest value of the applied potential a behavior similar to an asymmetric target pattern is observed (Fig. 6f): A front-like excitation travels once around the ring before it is extinguished again at a certain position. However, unlike in Fig. $6 \mathrm{~b}$ the behavior is no longer strictly periodic, and once in a while the wave emerges at a different position, survives the first rotation, and is extinguished only when it approaches the 'critical position' for the second time. In Fig. 6f this is, e.g., the case for the last pulse displayed.

\section{Discussion}

The patterns observed at large and small distance between the WE and the RE emerge in fundamentally different spatial instabilities, which can be attributed to the fact that in the first case the different positions of the electrode are coupled exclusively by migration currents, while for a close reference electrode, there is an additional global constraint present.

To make the different bifurcations involved most transparent, consider eqn. (1) and (2). They represent a general, dimensionless model for the spatio-temporal dynamics of an electrochemical oscillator under potentiostatic control in the case where the RE is far away from the WE such that we can exclude any global coupling. ${ }^{35,36}$

$$
\begin{gathered}
\frac{\partial \phi_{\mathrm{DL}}(x)}{\partial t}=f\left(\phi_{\mathrm{DL}}(x), c(x)\right)-\frac{\sigma}{\beta}\left(\left.\frac{\partial \phi(x)}{\partial z}\right|_{z=\mathrm{WE}}+\left(U-\phi_{\mathrm{DL}}(x)\right)\right) \\
\frac{\partial c(x)}{\partial t}=g\left(\phi_{\mathrm{DL}}(x), c(x)\right)
\end{gathered}
$$

$U$ is the externally applied voltage, $\sigma$ the specific conductivity of the electrolyte, which together with the geometric factor $\beta$ determines the uncompensated cell resistance per unit electrode area. $x$ and $z$ are the coordinates parallel to the (one-dimensional) electrode and normal to the electrode, respectively, $\phi$ the potential in the electrolyte and $z=\mathrm{WE}$, a position at the working electrode.

The two variables $\phi_{\mathrm{DL}}$ and $c$ are the potential drop across the double layer, the central variable for the description of electrode reactions, and the concentration (or coverage) of a chemical species, respectively. The functions $f$ and $g$ define the homogeneous dynamics of the system, which is of the activator-inhibitor type, whereby $\phi_{\mathrm{DL}}$ takes on the role of the activator and $c$ that of the inhibitor. Spatial coupling due to diffusion of $c$ is much smaller than migration coupling, and can thus be neglected.

The second term on the right-hand side of eqn. (1) represents migration currents that are induced by an inhomogeneous distribution of the double layer potential. ${ }^{2,37}$ Thus, it describes the spatial coupling between different locations of the electrode owing to an inhomogeneous potential distribution, and it is the counterpart of diffusion in reaction-diffusion systems. Just as does diffusion coupling, migration coupling acts in a synchronizing manner, i.e. in the absence of a reaction term it smoothes any spatial inhomogeneities. Note that in order to solve eqn. (1) it is necessary to 
determine the electric field normal to the electrode, which requires the solution of Laplace's equation $\Delta \phi=0$. This equation brings the directions parallel to the electrode into play, and it becomes clear why eqn. (1), which does not explicitly contain a spatial operator that depends on $x$, is capable of describing pattern formation along the electrode: the spatial coupling among different sites of the electrode is mediated through the bulk electrolyte. An inhomogeneous distribution of $\phi_{\mathrm{DL}}(x)$ changes the potential distribution, $\phi(x, y, z)$, in the entire electrolyte, and thus also the electric field at the WE, $\left.[\partial \phi(x) / \partial z]\right|_{z=W E}$.

In Fig. 3, i.e. for a large distance between the WE and the RE, the dynamics is dominated by the homogeneous mode which oscillates in time. Spatial structures pop up predominantly on the flanks of the oscillations when the homogeneous mode changes quickly, the amplitude of the inhomogeneous part of the oscillation being at most $50 \%$ of the amplitude of the uniform oscillation. This is a strong hint that the oscillations also exist in an infinitely small, 'point-like system'. In other words, we can view our system as being composed of (infinitely many) oscillatory elements coupled by migration currents.

It is apparent that the homogeneous oscillation is unstable with respect to large wavelength perturbations. This observation allows us to deduce the qualitative run of the real part of the largest Floquet multiplier $m$ as a function of the wavenumber of a spatial perturbation, $n$ (Fig. 7a). The homogeneous limit cycle is stable with respect to homogeneous perturbations and neutrally stable upon phase displacements. Thus, $m=1$ for $n=0$. In contrast, $m$ lies outside the unit circle for perturbations with a large wavelength, i.e. within an interval $n \in\left[0, n_{\mathrm{c}}\right]$ (see also ref. 38). For our later discussion it is useful to recall how the growth rate of perturbations of the homogeneous fixed point changes with the wavenumber of the perturbations (Fig. 7b). It is largest and positive for $n=0$, and decreases monotonically with increasing $n$, reflecting the oscillating nature of the local elements and the synchronizing spatial coupling on the activator variable.

Pattern formation in oscillating media coupled by diffusion has been studied extensively. ${ }^{39-42}$ Many studies were performed with the complex Ginzburg-Landau equation (CGLE) which is valid close to a Hopf bifurcation. The CGLE has the particular advantage that the stability of the homogeneous oscillation that is born in the Hopf bifurcation can be directly read off from the coefficients entering the equation. It was shown that if the so-called 'phase diffusion coefficient' is negative, the homogeneous oscillation is unstable with respect to spatial perturbations. For large systems, the long-term behavior is then typically an irregular turbulent motion. Such oscillating media have been called Benjamin-Feir unstable systems. ${ }^{41,43}$

According to the discussion in the second last paragraph, it appears obvious that the spatial structure in Fig. 3 arises because of a negative phase diffusion coefficient. Thus, we can classify our electrochemical oscillator as being Benjamin-Feir unstable, as was also done by Christoph for solutions of a reaction-migration system describing Ni dissolution. ${ }^{38}$ In contrast to most studies of Benjamin-Feir unstable systems, in our experiment the system length is of the same order of magnitude as the characteristic length of the pattern, and instead of 'electrochemical turbulence', regular limit cycle oscillations established. One should also keep in mind that (1) the oscillations are relaxation-like and (2) that they are of the mixed mode type. The fact that the small amplitude
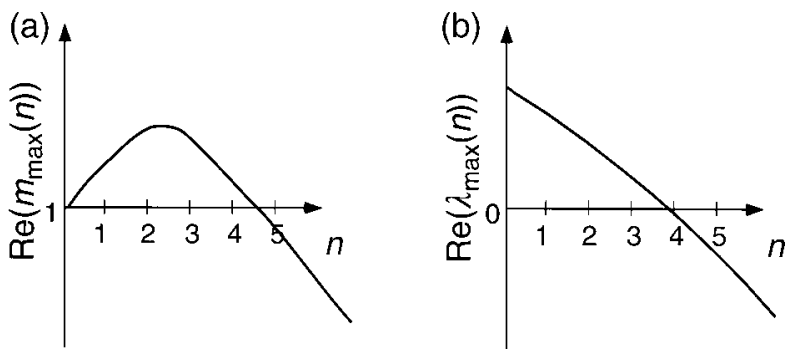

Fig. 7 (a) Schematic of the real part of the largest Floquet multiplier $\operatorname{Re}\left(m_{\max }(n)\right)$ as a function of the wavenumber $n$ of a perturbation of the homogeneous limit cycle. (b) Dispersion relation displaying the growth rate $\operatorname{Re}\left(\lambda_{\max }(n)\right)$ of perturbations of the homogeneous stationary state vs. their wavenumber $n$. (a) and (b) apply to the case of the limit cycle oscillations of Fig. 3. Note that the Floquet multiplier is real, i.e. $\operatorname{Im}\left(m_{\max }(n)\right)=0$ for the interesting range of $n$, whereas the largest eigenvalue $\lambda_{\max }(n)$ is complex for $0 \leqslant n \leqslant n_{\mathrm{c}}$. 
spiraling out of the trajectory is not accompanied by spatial structures ( $c f$. Fig. 3) strongly suggests that the mixed-mode character arises from an instability of the homogeneous system and not from spatial instabilities. Hence, the proper description of the data of Fig. 3 requires a 3-component reaction-migration system, and the oscillations are far from a supercritical Hopf bifurcation. Both instances restrict further comparison of the experimental behavior with solutions of the CGLE and call for additional theoretical studies that take into account these complications. We are currently developing an extended ordinary differential equation (ODE) system in order to describe the more complex temporal behavior. However, besides this realistic modeling, a more general, 'normal form-type' approach would also be desirable.

Benjamin-Feir unstable states were observed in hydrodynamical systems. ${ }^{43-46}$ Numerical studies found that most reaction-diffusion systems also possess a Benjamin-Feir unstable region. However, corresponding experimental examples in reaction-diffusion type systems are rare. Turbulent states that might be associated with a negative effective phase diffusion coefficient were reported for $\mathrm{CO}$ oxidation on $\mathrm{Pt}(110)^{47,48}$ and for the $\mathrm{NO}+\mathrm{NH}_{3}$ reaction on $\mathrm{Pt}(100),{ }^{49}$ both under low pressures. Further analysis of these turbulent states that would allow an unambiguous assignment of the dynamical state of the system has not yet been reported.

Now, let us turn to a discussion of the dynamics found for the closest distance between the WE and the RE. This geometry introduces a negative global coupling into the system which manifests itself in an additional term in eqn. (1) that depends on the average double layer potential, $\left\langle\phi_{\mathrm{DL}}\right\rangle_{x}$ :

$$
\frac{\partial \phi_{\mathrm{DL}}(x)}{\partial t}=f\left(\phi_{\mathrm{DL}}(x), c\right)+\alpha\left(\phi_{\mathrm{DL}}(x)-\left\langle\phi_{\mathrm{DL}}\right\rangle_{x}\right)-\frac{\sigma}{\beta}\left(\left.\frac{\partial \phi}{\partial z}\right|_{z=\mathrm{WE}}+\left(U-\phi_{\mathrm{DL}}(x)\right)\right)
$$

where $\alpha>0$ is a parameter that depends on the difference of the resistances between the WE and the RE, on the one hand, and the WE and the CE, on the other. It determines the strength of the global coupling. ${ }^{28}$ Qualitatively, the global coupling in eqn. (3) acts in a destabilizing manner: If at a certain position $x_{1}, \phi_{\mathrm{DL}}\left(x_{1}\right)$ is larger than the average double layer potential, the global coupling term (the second term on the right-hand side in eqn. (3)) is positive and thus causes a further increase of $\phi_{\mathrm{DL}}\left(x_{1}\right)$. If, on the other hand, $\phi_{\mathrm{DL}}\left(x_{1}\right)$ is smaller than $\left\langle\phi_{\mathrm{DL}}\right\rangle_{x}$, the term is negative and again the negative perturbation at $x_{1}$ is enhanced. Such type of global coupling has been coined negative global coupling (because of the negative sign in front of $\left\langle\phi_{\mathrm{DL}}\right\rangle_{x}$ ). A different sign in front of the global coupling term turns the action of the coupling into a synchronizing one, which is also called a positive global coupling.

To recall the influence of the global coupling on the stability of a stationary state, consider two systems that possess identical homogeneous states, but one system experiences a global constraint, the other one not. In the case of our electrochemical system, for example, we can prepare these states by changing the position of the CE. If the CE is at the same height as the RE, $\alpha$ in eqn. (3) is 0 and the system is only coupled by migration coupling. If we put the CE further away than the RE, the stationary states remain identical but global coupling comes into play. Since the global coupling affects only the homogeneous mode (through the average double layer potential), the two systems exhibit the same dependence of the largest eigenvalue of the linearized system $\lambda_{\max }$, on the wavenumber of the perturbation, $n$, for $n>0$. But the respective eigenvalues differ for $n=0$. If the average double layer potential enters into the activator equation with a negative sign, as in eqn. (3), it is straightforward to deduce that the homogeneous mode is stabilized and the dispersion relation possesses the qualitative shape shown in Fig. 8. In particular, for strong negative coupling or large systems $\lambda_{\max }(n=1)$ will be larger than $\lambda_{\max }(n=0)$. This means that when starting from a stable stationary state with only negative real parts of all eigenvalues and changing a parameter such that the system is driven into an unstable region, the first eigenvalue to become unstable is the one with $n=1$. If the eigenvalue is real, this bifurcation gives rise to stationary domains, as were observed in nonisothermic catalytic reactions, ${ }^{4,50}$ during the electrochemical reduction of peroxodisulfate ${ }^{28}$ or also during the oxidation of $\mathrm{H}_{2}$ on $\mathrm{Pt}$ for different electrolyte concentrations and compositions than used here. ${ }^{3,29}$ For complex eigenvalues, a wave bifurcation occurs. Owing to the rotational invariance of our 1-dimensional spatial domain, the eigenfunctions of the system are Fourier modes whereby sine and cosine modes are degenerate, and become unstable simultaneously. Hence, for initial perturbations with predominantly even or odd symmetry, standing waves with wavenumber one might develop (i.e. only the sine, or equivalently the cosine is 


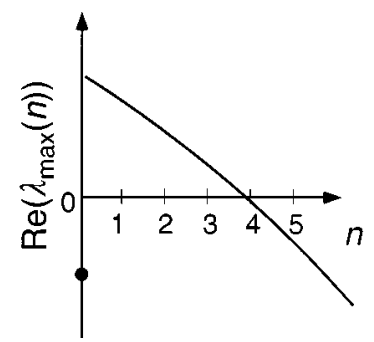

Fig. 8 Schematic dispersion relation displaying the growth rate $\operatorname{Re}\left(\lambda_{\max }(n)\right)$ of perturbations of the homogeneous stationary state $v$ s. the wavenumber $n$ of the perturbation in the case of negative (desynchronizing) global coupling. This situation applies to the data shown in Fig. 5 and 6. Note that $\lambda_{\max }(n)$ is complex for $0 \leqslant n \leqslant n_{\mathrm{c}}$.

excited). Such waves were observed, e.g., during formic acid oxidation on a Pt ring-electrode when the RE was put sufficiently close to the WE. ${ }^{30}$ For all other perturbations, in which $\sin (x)$ and $\cos (x)$ are both excited sufficiently, travelling waves or phase pulses prevail.

We thus conclude that the pulses we observed for a close reference electrode ( $c f$. Fig. 5) are associated with a wave bifurcation of the stationary state with $n=1$. In contrast to the patterns observed with the external RE, that arose due to a spatial instability of the homogeneous limit cycle, here we are dealing with a non-trivial Hopf bifurcation of the homogeneous stationary state.

The experimental pulses were not at any time 'perfect' in the sense that they possessed a strictly constant shape or velocity ( $c f$. Fig. 5). This can already be deduced from the time series shown in Fig. 4, which is oscillatory with a period that corresponds to the time in which the pulse travels once around the ring. A constant shape would result in a stationary current, a constant velocity in addition in a stationary domain in the moving frame. Instead, in the moving frame the experimental pulses displayed a zigzag motion, reflecting a variation in the speed of the pulse, superimposed by a minor alteration of the width. This behavior might be the manifestation of secondary bifurcations of the pulse, such as an oscillatory instability that leads to variations in width.

However another interpretation appears to be more likely: An electrode surface, like any catalytic surface, is never an ideally homogeneous medium. Rather, its reactivity is likely to differ somewhat from position to position, and it might also change with time. Thus, the pulse might experience different local properties while propagating around the electrode. These inhomogeneities will in general affect pulse width and velocity. The fact that the periods of the oscillations in width in Fig. $5 \mathrm{~b}$ or $\mathrm{d}$ coincide with the rotation period of the pulse supports this conjecture (although a $1: 1$ locking of the two frequencies also cannot be excluded a priori).

Provided that the fine structure of the pulses stems from nonhomogeneities of the electrode surface, the sequence of time series shown in Fig. 4 suggests that the interaction between the intrinsic dynamics and the nonhomogeneous distribution of parameters is very intricate. This points to a possible control of the dynamics by deliberately designing catalysts with spatially modulating properties. In the electrochemical context, e.g., the poisoning of the electrode by the reaction intermediate $\mathrm{CO}$ is the major problem in all oxidation reactions of small organic molecules. Their most important application is in fuel cells, such as the direct methanol fuel cell. ${ }^{51}$ All these oxidation reactions exhibit oscillatory behavior and they belong to the same class of electrochemical oscillators as the system studied here. If it could be shown that the interaction between a nonuniform activity of the electrode and the nonlinear reaction dynamics can prevent complete poisoning of the catalytic surface at low overvoltage, this would be a major breakthrough in fuel cell research. Progress along these lines will not be possible without theoretical foundations. Work in this direction has begun, ${ }^{52-56}$ and much more is needed to reach the goal of controlling patterns and overall reaction rates by the nonuniformity of active media.

In the case of intermediate distance between the WE and the RE, the strength of the global coupling is smaller than in the case of the close RE which apparently enables more diverse pattern formation.

Target patterns were found in simulations of an electrochemical oscillator with Benjamin-Feir stable homogeneous limit cycles and negative global coupling. ${ }^{38}$ Because of the similarity between 
target patterns and asymmetric target patterns we believe that the patterns observed in Fig. $6 \mathrm{~b}-\mathrm{f}$ emerge owing to the global constraint and do not require a Benjamin-Feir unstable limit cycle. On the other hand, the spatio-temporal behavior of Fig. 6a appears to be of a different quality and we speculate that the large amplitude bursts in Fig. 6a might be linked to the fact that the homogeneous oscillation without global coupling is Benjamin-Feir unstable. Hence, in this case, the spatio-temporal pattern would exist only in Benjamin-Feir unstable systems that are subject to a negative global coupling. To substantiate this conjecture, model calculations are required, that extend those on pattern formation in the CGLE with global coupling, ${ }^{14,22,57,58}$ in which the corresponding behavior has not been found. Furthermore, we have discussed that in the absence of global coupling, the homogeneous dynamics can only be described by a set of three essential variables (a three-component system). As long as the dynamics of Fig. 6a (and also 6d) is not further characterized, we cannot exclude that these complex motions are the result of the interaction of three variables.

\section{Conclusions}

We presented experiments on an electrochemical oscillator subject to global, desynchronizing coupling with varying strength. The experiments revealed several novel aspects that broaden our view on pattern formation in the presence of nonlocal constraints, and they point to directions in which further theoretical foundations are needed. Among them are (1) Pattern formation in Benjamin-Feir unstable systems: (a) in which the system size and characteristic size of the patterns are of the same order of magnitude, (b) close to a subcritical or homoclinic bifurcation, (c) in three-component reaction-transport models and (d) with an additional desynchronizing global constraint. (2) A more detailed understanding of how the strength of the global coupling affects possible bifurcation scenarios and the diversity of dynamical states. (3) Pattern formation in systems with distributed parameters, in particular with emphasis on tailoring inhomogeneous active media to obtain catalytic surfaces with improved performance.

\section{Acknowledgements}

We are grateful to G. Ertl for stimulating interest in this work and for generous support and thank A. Bonnefont, F. Plenge, J. Christoph and M. Eiswirth for fruitful discussions. A grant from the DFG in the framework of the Sfb555 is gratefully acknowledged.

\section{References}

1 M. T. M. Koper, in Advances in Chemical Physics, ed. I. Prigogine and S. A. Rice, Wiley, New York, 1996, vol. 92 , p. 161.

2 K. Krischer, in Modern Aspects of Electrochemistry, ed. B. E. Conway, J. O. M. Bockris and R. White, Kluwer Academic/Plenum, New York, 1999, vol. 32, p. 1.

3 K. Krischer, N. Mazouz and P. Grauel, Angew. Chem. Int. Ed., 2001, 40, 851.

4 Y. E. Volodin, V. V. Barelko and A. G. Merzhanov, Sov. J. Chem. Phys., 1982, 5, 1146.

5 G. Philippou and D. Luss, Chem. Eng. Sci., 1993, 48, 2313.

6 U. Middya, M. D. Graham, D. Luss and M. Sheintuch, Physica D, 1993, 63, 393.

7 M. D. Graham, S. L. Lane and D. Luss, J. Phys. Chem., 1993, 97, 7564.

8 R. Imbihl and G. Ertl, Chem. Rev., 1995, 95, 697.

9 H. Willebrand, T. Hüntler, F. J. Niedernostheide, R. Dohmen and H.-G. Purwins, Phys. Rev. A, 1992, 45, 8766.

10 E. Schöll, Nonlinear Spatio-Temporal Dynamics and Chaos in Semiconductors, Cambridge University Press, Cambridge, 2001.

11 F. J. Elmer, Physica D, 1988, 30, 321.

12 M. Sheintuch, AIChE J., 1989, 42, 1081.

13 U. Middya, M. D. Graham, D. Luss and M. Sheintuch, J. Chem. Phys., 1993, 98, 2823.

14 M. Falcke, H. Engel and M. Neufeld, Phys. Rev. E, 1995, 52, 763.

15 M. Falcke and H. Engel, Phys. Rev. E, 1997, 56, 635.

16 A. Alekseev, S. Bose, P. Rodin and E. Schöll, Phys. Rev. E, 1998, 57, 2640.

17 S. Bose, P. Rodin and E. Schöll, Phys. Rev. E, 2000, 62, 1778.

18 M. Meixner, P. Rodin and E. Schöll, Phys. Rev. E, 1998, 58, 5586.

19 M. Meixner, P. Rodin and E. Schöll, Phys. Rev. E, 1998, 58, 2796. 
20 F.-J. Niederostheide, R. Dohmen, H. Willebrand, B. S. Kerner and H.-G. Purwins, Physica D, 1993, 69, 425.

21 D. Battogtokh, M. Hildebrand, K. Krischer and A. S. Mikhailov, Phys. Rep., 1997, 288, 435.

22 F. Mertens, R. Imbihl and A. Mikhailov, J. Chem. Phys., 1994, 101, 9903.

23 K. C. Rose, D. Battogtokh, A. S. Mikhailov, R. Imbihl, W. Engel and A. M. Bradshaw, Phys. Rev. Lett., 1996, 76, 3582 .

24 I. Z. Kiss, W. Wang and J. L. Hudson, Phys. Chem. Chem. Phys., 2000, 2, 3847.

25 R. D. Otterstedt, P. J. Plath, N. I. Jaeger and J. L. Hudson, J. Chem. Soc., Faraday Trans., 1996, 92, 2933.

26 R. D. Otterstedt, N. I. Jaeger, P. J. Plath and J. L. Hudson, Chem. Eng. Sci., 1999, 54, 1221.

7 J. Christoph, R. D. Otterstedt, M. Eiswirth, N. I. Jaeger and J. L. Hudson, J. Chem. Phys., 1999, 110, 8614.

P. Grauel, J. Christoph, G. Flätgen and K. Krischer, J. Phys. Chem. B, 1998, 102, 10264.

P. Grauel and K. Krischer, Phys. Chem. Chem. Phys., 2001, 3, 2497.

P. Strasser, J. Christoph, W.-F. Lin, M. Eiswirth and J. L. Hudson, J. Phys. Chem. B, 2000, 104, 1854.

J. Christoph, P. Strasser, M. Eiswirth and G. Ertl, Science, 1999, 284, 291.

K. Krischer, N. Mazouz and G. Flätgen, J. Phys. Chem., 2000, 104, 7545.

H. Varela and K. Krischer, Catal. Today, 2001, in press.

A. Zhabotinsky, M. Dolnik and I. Epstein, J. Chem. Phys., 1995, 103, 10306.

G. Flätgen and K. Krischer, J. Chem. Phys., 1995, 103, 5428.

6 N. Mazouz, K. Krischer, G. Flätgen and G. Ertl, J. Phys. Chem., 1997, 101, 2403.

7 N. Mazouz, G. Flätgen and K. Krischer, Phys. Rev. E, 1997, 55, 2260.

J. Christoph, PhD Thesis, FU Berlin, Berlin, Germany, 1999.

39 M. C. Cross and P. C. Hohenberg, Rev. Mod. Phys., 1993, 65, 851.

Y. Kuramoto, Chemical Oscillations, Waves and Turbulence, Springer, Berlin, 1984

1 A. S. Mikhailov, Foundations of Synergetics I, Springer, Berlin, 1994.

42 A. S. Mikhailov and A. Y. Loskutov, Foundations of Synergetics II, Springer, Berlin, 1996.

43 T. Benjamin and J. Feir, J. Fluid Mech., 1967, $27,417$.

44 B. Lake and H. Yuen, J. Fluid Mech., 1977, 83, 75.

45 Y. Liu and R. E. Ecke, Phys. Rev. Lett., 1997, 78, 4391.

46 W. K. Melville, J. Fluid Mech., 1982, 115, 165.

47 S. Jakubith, H. H. Rotermund, W. Engel, A. v. Oertzen and G. Ertl, Phys. Rev. Lett., 1990, 65, 3013.

48 M. Kim, M. Bertram, M. Pollmann, A. v. Oertzen, A. S. Mikhailov, H. H. Rotermund and G. Ertl, Science, 2001, 292, 1357.

49 G. Veser, F. Esch and R. Imbihl, Catal. Lett., 1992, 13, 371.

50 L. Lobban, G. Philippou and D. Luss, J. Chem. Phys., 1991, 93, 733.

51 L. Carrette, K. A. Friedrich and U. Stimming, ChemPhysChem, 2000, 1, 162.

52 A. K. Bangia, M. Bär, I. G. Kevrekidis, M. D. Graham, H.-H. Rotermund and G. Ertl, Chem. Eng. Sci., 1996, 51, 1757

53 A. Kulka, M. Bode and H.-G. Purwins, Phys. Lett. A, 1995, 203, 33.

54 A. Hagberg, E. Meron, I. Rubinstein and B. Zaltsman, Phys. Rev. Lett., 1996, 76, 427.

55 M. A. Liauw, J. Ning and D. Luss, J. Chem. Phys., 1996, 104, 5657.

56 S. Y. Shvartsman, E. Schütz, R. Imbihl and I. G. Kevrekidis, Phys. Rev. Lett., 1999, 83, 2857.

57 D. Battogtokh, A. Preusser and A. S. Mikhailov, Physica D, 1997, 106, 327.

58 D. Lima, D. Battogtokh, A. S. Mikhailov, P. Borckmans and G. Dewel, Europhys. Lett., 1998, 42, 631. 